\title{
RR Lyrae stars as tracers of halo substructures
}

\author{
Sonia Duffau ${ }^{1,2, \star}$, A. Katherina Vivas ${ }^{3}$, Camila Navarrete ${ }^{2,1}$, Julio Carballo-Bello ${ }^{2,1}$, Gergely \\ $\mathrm{Hajdu}^{2,1}$, and Márcio Catelann ${ }^{2,1}$ \\ ${ }^{1}$ Millennium Institute of Astrophysics, Santiago, Chile \\ ${ }^{2}$ Pontificia Universidad Católica de Chile, Instituto de Astrofísica, Av. Vicuña Mackenna 4860, 782-0436 \\ Macul, Santiago, Chile \\ ${ }^{3}$ Cerro Tololo Interamerican Observatory, Casilla 603, La Serena, Chile
}

\begin{abstract}
Three RR Lyrae overdensity candidates in the southern sky have been studied using low resolution spectra obtained with the Goodman spectrograph at SOAR. We search for unidentified velocity peaks indicative of the possible presence of streams within the overdensities. Our results suggest that all three overdensities present an excess of radial velocity signal at high mean radial velocities which cannot be explained as a contribution from any known Galactic component.
\end{abstract}

\section{Introduction}

We study halo substructure to determine how important a role accretion has played in the formation and evolution of our Galaxy. We do so by using RR Lyrae as tracers, because they are old and bright, and excellent standard candles.

A large effort has been conducted already unveiling substructure in the northern sky, while the southern sky census is still fairly incomplete. From the catalog of RR Lyrae stars presented in [4] we targeted three overdensity candidates. The overdensities in this study are: Hya 2, Crv 1 and Cen 3, having 28, 15 and 20 RR Lyrae members each and with mean heliocentric distances of 9.4, 11.4 and $10.2 \mathrm{kpc}$, respectively. From low resolution spectra of the member stars, radial velocities have been obtained by cross-correlation with appropriate templates, and the pulsation velocity of these variable stars has been accounted for. As a by-product, mean metallicities have been obtained as well, from the equivalent widths of the Ca II K line and three Balmer lines using Layden's 1994 method ([3]).

\section{Observations and results}

After obtaining all velocities, we compare the resulting histograms to the expected velocity distribution for the potential galactic contaminants, and check possible associations between our overdensities and previous discoveries in the area. For all three overdensities an excess of high velocity radial velocity signal was found. Figure 1 displays, as an example, the results for Crv 1 . In the left panel, the radial velocity histogram (smoothed with a window comparable to the radial velocity error) of the data is presented (in black), along with that for model particles of the Galaxy obtained from the Besançon

^sduffau@astro.puc.cl 


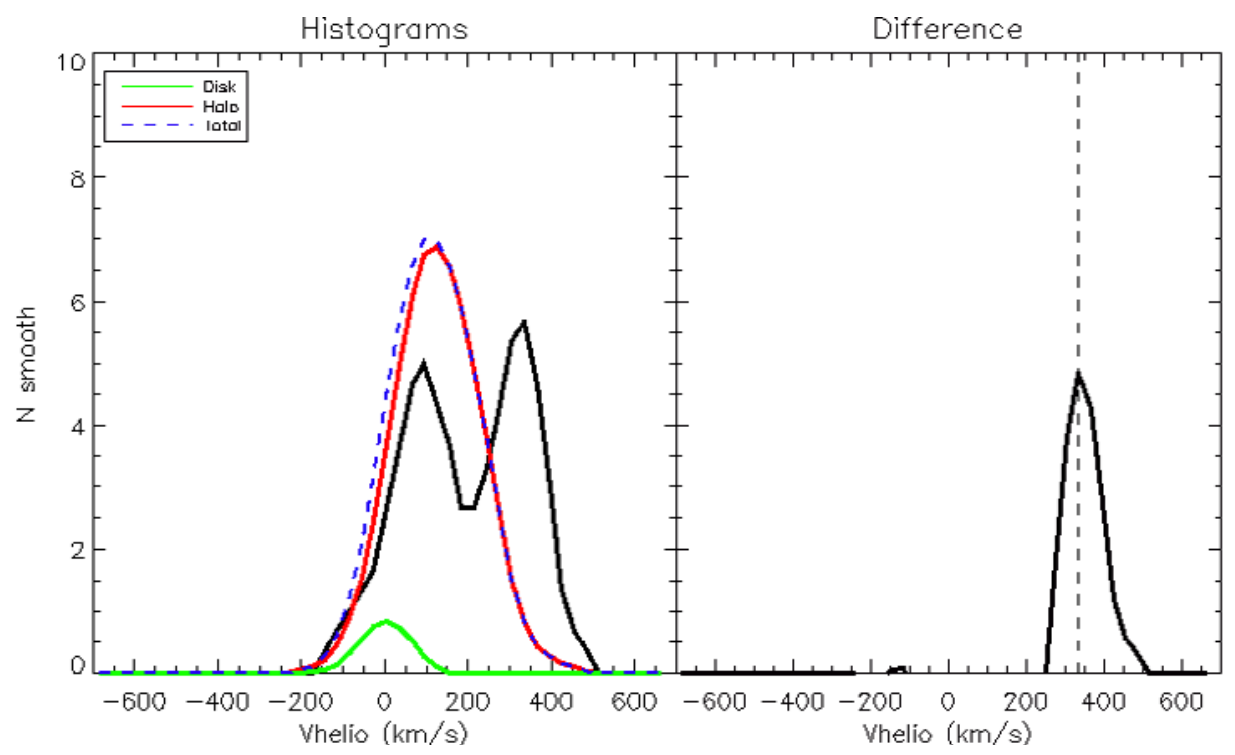

Figure 1. Radial velocity distribution obtained for Crv 1 (black solid line). The dashed blue line indicates the velocity distribution of the Galaxy as predicted by the Besançon model for the same line-of-sight (composed of a halo contribution, in red, and a disk contribution, in green). The vertical line indicates the position of the detected excess signal. Equivalent results were found for Cen 3 and Hya 2 at very similar mean peak velocities.

model for the location of Crv 1 (in red and green). Figure 1 also displays the total contribution from the Galaxy (as a dashed blue line). The potential Galaxy contaminants considered belong to the halo and thick disk population, depicted as red and green smoothed histograms. In the right panel, the excess signal in radial velocity of Crv 1 is displayed. The excess is defined as the difference between the data and the total contribution from the Galaxy, and indicates the presence of a high velocity peak not explained as part of the Milky Way. We interpret this as the original signal from the overdensity, and as evidence of a potential stream candidate.

All three overdensities present an excess of radial velocity signal at high mean radial velocities, as exemplified in Figure 1, which cannot be explained as a contribution from any known Galactic component. Hya 2 is a new detection. Crv 1 seems to be the southern extension to the Virgo Stellar Stream and G4 velocity complex discovered in [5]. Although at a similar location as the expected orbit of the Orphan Stream, Cen 3's distance and velocity do not correspond with the expected values in the area, representing then another new detection as well. Confirmation of the results will be obtained by applying a group finding algorithm to the data, considering both the radial velocity of the stars and the separation between them, and simulating the Galactic contribution to assess the significance of these detections, as done in [1] and [5].

Acknowledgments: We acknowledge financial support from the Ministry for the Economy, Development, and Tourism's Programa Iniciativa Científica Milenio through grant IC 120009, awarded to the Millennium Institute of Astrophysics (MAS), from CONICYT's PCI program through grant DPI20140066, from Proyecto Basal CATA PFB-06/2007, and from FONDECYT grants \#1171273 and \#3160502. 


\section{References}

[1] Duffau, S., Vivas, A. K., Zinn, R., Méndez, R. A., \& Ruiz, M. T., A\&A, 566, A118 (2014)

[2] Grillmair, C. J., Hetherington, L., Carlberg, R. G., \& Willman, B., ApJL, 812, L26 (2015)

[3] Layden, A. C., AJ, 108, 1016, (1994)

[4] Torrealba, G., Catelan, M., Drake, A. J., et al., MNRAS, 446, 2251 (2015)

[5] Vivas, A. K., Zinn, R., Farmer, J., Duffau, S., \& Ping, Y., ApJ, 831, 165 (2016) 